\title{
Determining the Psychophysiological Responses of Military Aircrew When Exposed to Acute Disorientation Stimuli
}

\author{
José Francisco Tornero Aguilera, J Gil-Cabrera, V J Clemente-Suárez
}

\begin{abstract}
Introduction: Exposure to enviromental flight conditions may impair performance and physical integrity, thus training in simulated environments it is a key factor. This research aimed to study the psychophysiological response, cortical arousal and autonomic modulation of pilots and medical aircrew personnel during disorientation exposure, considering gender, experience, flying hours and body mass index (BMI) as influencial variables. Methods: A total of 47 soldiers (37 men and 10 women, 22 medical aircrew personnel and 25 fighter pilots) of Spanish Air Forces faced 25 min of vestibular, proprioceptive and visual disorientation.

Results: Disorientation exposure elicited an increased psychophysiological response, significant increases in isometric hand strength, cortical arousal, autonomic modulation, perceived stress and effort in both groups while a significant decrease in respiratory muscle capacity and blood oxygen saturation in the medical aircrew group were found. Cross-sectional analysis showed gender differences, males presented greater parasympathetic activity and strength. Larger BMI was associated with greater levels and perception of stress as well as lower cardiovascular performance and sympathetic modulation. Furthermore, experience, previous training and larger flying hours correlated with greater parasympathetic modulation. Conclusion: Disorientation exposure produces an increase in cortical arousal and decrease in the parasympathetic nervous system either in pilots and medical aircrew personnel. In addition, medical aircrew personnel are less adapted to disorientation stimulus presenting significantly higher psychophysiological stress response, thus complementary physical training should be mandatory.
\end{abstract}

\section{Keywords:}

Education \& training; Physiology; Quality in health care. 